\title{
СХЕМА ЗАМЕЩЕНИЯ ДИФФЕРЕНЦИАЛЬНО- ТРАНСФОРМАТОРНОГО МАГНИТНОГО УСИЛИТЕЛЯ НА ДРОССЕЛЯХ С ПРЯМОУГОЛЬНОЙ КРИВОЙ НАМАГНИЧИВАНИЯ
}

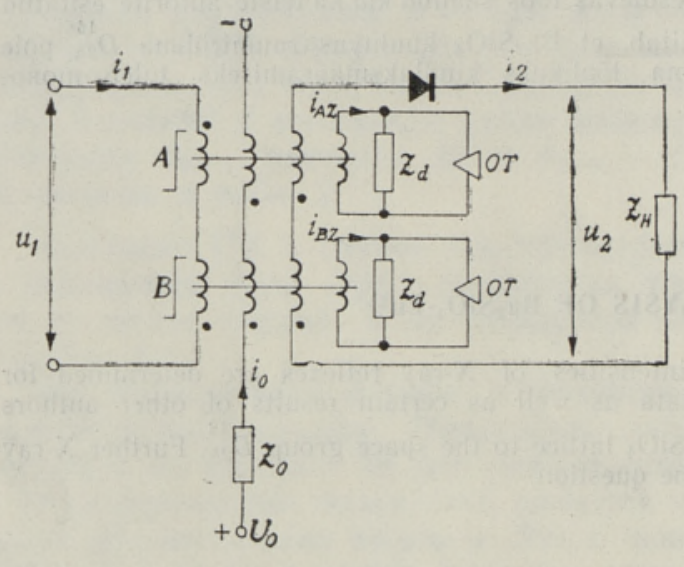

Рассмотрим приведенную на рис. 1 схему усилителя [ $\left.{ }^{1}\right]$. Обозначения следующие: $Z_{d}-$ динамическое сопротивление учитывает динамические свойства сердечника; OT - симметричный ограничитель тока - учитывает ширину гистерезисной петли $\left(2 i_{s}\right) ; u_{1}-$ напряжение питания; $U_{0}-$ э. д. с. управления; $Z_{0}-$ сопротивление в цепи управления; $Z_{H}-$ сопротивление нагрузки.

Қак видно, суммарные токи дросселей связаны системой уравнений

Рис. 1.

Предположим, что

$$
\begin{aligned}
& i_{A}=i_{1}+i_{0}+i_{2}-i_{A Z}, \\
& i_{B}=-i_{1}+i_{0}+i_{0}-i_{B Z} .
\end{aligned}
$$

откуда

$$
\begin{aligned}
& i_{A Z}=+i^{\prime}{ }_{1}-i^{\prime}{ }_{2}, \\
& i_{B Z}=+i^{\prime}{ }_{1}+i^{\prime}{ }_{2},
\end{aligned}
$$

Подставив (2) в систему (1), получим

$$
\begin{aligned}
& i^{\prime}{ }_{1}=\frac{i_{A Z}+i_{B Z}}{2}, \\
& {i^{\prime}}_{2}=\frac{i_{B Z}-i_{A Z}}{2} .
\end{aligned}
$$

где

$$
\begin{aligned}
& i_{A}=i^{\prime \prime}{ }_{1}+i^{\prime \prime}{ }_{2}+i_{0}, \\
& i_{B}=-i^{\prime \prime}{ }_{1}+i^{\prime \prime}{ }_{2}+i_{0},
\end{aligned}
$$

$$
\begin{aligned}
& i^{\prime \prime}{ }_{1}=i_{1}-i^{\prime}{ }_{1}, \\
& i^{\prime \prime}{ }_{2}=i_{2}+i^{\prime}{ }_{2} .
\end{aligned}
$$


Так как

$$
\begin{aligned}
& i_{A Z}=\frac{u_{A}}{Z_{d}}+i_{s}, \\
& i_{B Z}=\frac{u_{B}}{Z_{d}}+i_{s},
\end{aligned}
$$

To

$$
i_{1}^{\prime}=\frac{\frac{u_{A}-1-u_{B}}{Z_{d}}+2 i_{s}}{2}=\left(\frac{u_{1}}{2 Z_{d}}+i_{s}\right) \text {, }
$$

$$
i_{2}^{\prime}=\frac{u_{B}-u_{A}}{2 Z_{d}}=\frac{u_{2}}{2 Z_{d}}
$$

Системе уравнений (4) соответствует схема рис. 2. Пренебрегая $i_{1}^{\prime}$, так как он не влияет на режим работы усилителя, и совмещая обмотки управления и выхода, так как они соединены одинаково, приходим к схеме замещения на рис. 3.

Обозначим эквивалентное сопротивление двухполюсни-

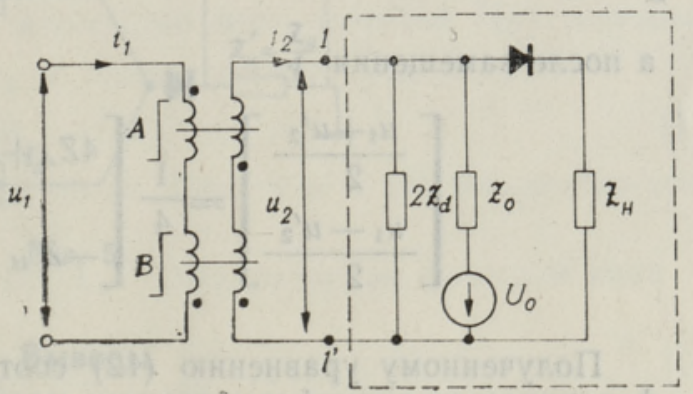

Рис. 3. ка (11') через $Z_{H}^{\prime}$, эквивалентную э.д. с. через $u_{2}^{\prime}$ и индуктивные сопротивления взаимной индукции обмоток дросселей соответственно через $Z_{A}=M_{A} d / d t$ и $Z_{B}=M_{B} d / d t$ и запишем отвечающее схеме рис. 3 матричное уравнение

$$
\left[\begin{array}{c}
u_{1} \\
u_{2}^{\prime}
\end{array}\right]=\left[\begin{array}{cc}
Z_{A}+Z_{B} & Z_{A}-Z_{B} \\
Z_{A}-Z_{B} & Z^{\prime}{ }_{H}+Z_{A}+Z_{B}
\end{array}\right]\left[\begin{array}{c}
i_{1} \\
i_{2}
\end{array}\right]
$$


где

$$
\begin{aligned}
& Z_{A}+Z_{B}=a_{11}, \quad Z_{A}+Z_{B}+Z_{H}^{\prime}=a_{22} ; \\
& Z_{A}-Z_{B}=a_{12}=a_{21},
\end{aligned} \quad\left[\begin{array}{ll}
a_{11} & a_{12} \\
a_{21} & a_{22}
\end{array}\right]=A .
$$

Дроссельные токи $i_{A}$ и $i_{B}$ представим в виде

$$
\left[\begin{array}{l}
i_{A} \\
i_{B}
\end{array}\right]=\left[\begin{array}{rr}
1 & 1 \\
1 & -1
\end{array}\right]\left[\begin{array}{l}
i_{1} \\
i_{2}
\end{array}\right]=C\left[\begin{array}{l}
i_{1} \\
i_{2}
\end{array}\right]
$$

Перейдя на новые координаты тока $\left[\begin{array}{l}i_{A} \\ i_{B}\end{array}\right]$, для соответствующих им напряжений получим выражение

$$
\left[\begin{array}{l}
u_{A} \\
u_{B}
\end{array}\right]=C_{t}^{-1}\left[\begin{array}{l}
u_{1} \\
u_{2}^{\prime}
\end{array}\right]=\frac{1}{2}\left[\begin{array}{rr}
1 & 1 \\
1 & -1
\end{array}\right]\left[\begin{array}{l}
u_{1} \\
u_{2}^{\prime}
\end{array}\right]=\left[\begin{array}{l}
\frac{u_{1}+u_{2}^{\prime}}{2} \\
\frac{u_{1}-u_{2}^{\prime}}{2}
\end{array}\right]
$$

и для соответствующей матрицы сопротивления - выражение

$$
\begin{aligned}
& A^{\prime}=C_{t}^{-1} A C^{-1}=\frac{1}{4}\left[\begin{array}{rr}
1 & 1 \\
1 & -1
\end{array}\right]\left[\begin{array}{ll}
a_{11} & a_{12} \\
a_{21} & a_{22}
\end{array}\right]\left[\begin{array}{rr}
1 & 1 \\
1 & -1
\end{array}\right]= \\
= & \frac{1}{4}\left[\begin{array}{ll}
a_{11}+a_{21}+a_{12}+a_{22} & a_{11}+a_{21}-a_{12}-a_{22} \\
a_{11}-a_{21}+a_{12}-a_{22} & a_{11}-a_{21}-a_{12}+a_{22}
\end{array}\right] .
\end{aligned}
$$

Итак:

$\left[\begin{array}{c}\frac{u_{1}+u_{2}^{\prime}}{2} \\ \frac{u_{1}-u_{2}^{\prime}}{2}\end{array}\right]=\frac{1}{4} \cdot\left[\begin{array}{ll}a_{11}+a_{21}+a_{12}+a_{22} & a_{11}+a_{21}-a_{12}-a_{22} \\ a_{11}-a_{21}+a_{12}-a_{22} & a_{11}-a_{21}-a_{12}+a_{22}\end{array}\right]\left[\begin{array}{c}i_{A} \\ i_{B}\end{array}\right]$,

а после замещения

$$
\left[\begin{array}{c}
\frac{u_{1}+u_{2}^{\prime}}{2} \\
\frac{u_{1}-u_{2}^{\prime}}{2}
\end{array}\right]=\frac{1}{4}\left[\begin{array}{cc}
4 Z_{A}-Z_{H}^{\prime} & -Z_{H}^{\prime} \\
-Z_{H}^{\prime} & 4 Z_{A}+Z_{H}^{\prime}
\end{array}\right]\left[\begin{array}{l}
i_{A} \\
i_{B}
\end{array}\right]
$$

Полученному уравнению (12) соответствует схема замещения дифференциально-трансформаторного магнитного усилителя, показанная на рис. 4. Заменяя здесь $Z_{0} / 4$ и $U_{0} / 2$ источником тока $I_{0}$ вследствие большой линейной индуктивности $Z_{0}$, а также пренебрегая динамическим сопротивлением $Z_{d}$, получим схему, изображенную на рис. 5. Эта схема, как видно, эквивалентна симметричному блоку дизъюнкции (конъюнкции) на магнитных ключах с выпрямленным выходом $\left[{ }^{2,3}\right]$.

При этих упрощениях дифференциально-трансформаторный магнитный усилитель можно выразить аналитически в виде

$$
u_{H}^{\prime}(t)=0 \bigvee \frac{d}{d t}\left(\Psi_{11} \bigvee \Psi_{12}\right),
$$


где $\Psi_{1 k}-$ переменная составляющая фиктивного потокосцепления, соответствующая входному напряжению $u_{1 j}\left[{ }^{2}\right]$,

$$
\bigvee_{i=1}^{n} X_{i}=\max \left\{X_{i} \mid i=1, \ldots, n\right\}
$$

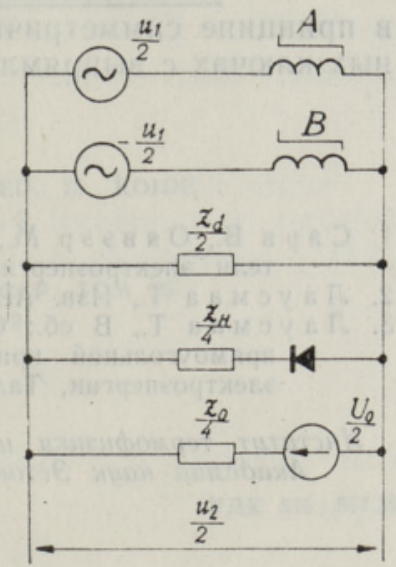

Рис. 4.
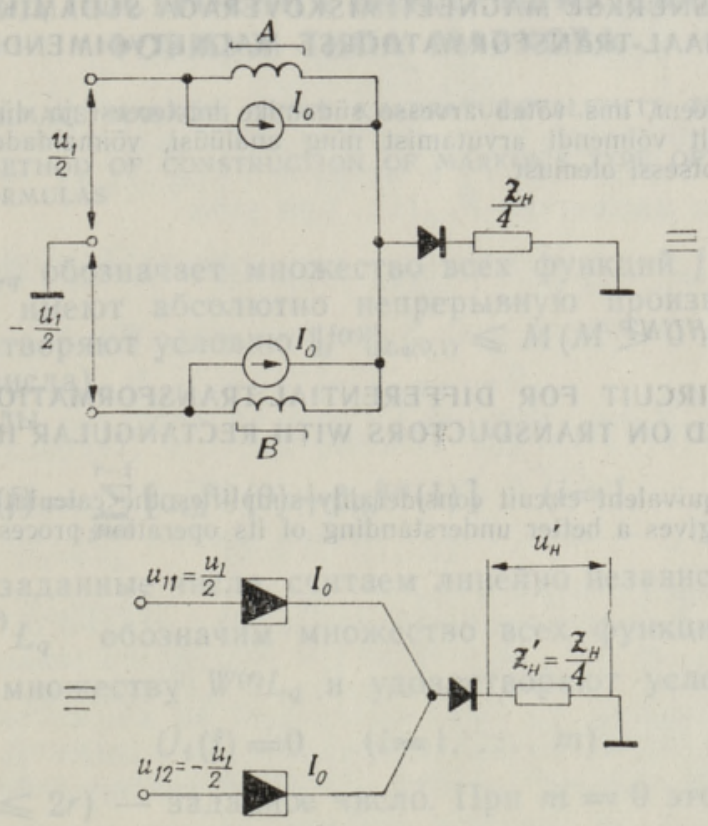

Pile. 5 .

\section{Выводы}

Предложенная схема замещения позволяет значительно упростить анализ и расчет усилителя, а также лучше понять процесс его работы, который заключается в следующем.

1. Ширина гистерезисной петли сердечников не влияет на режим работы усилителя.

2. Влияние динамического сопротивления на режим работы усилителя можно учесть с помощью соответствующего шунтирующего сопротивления.

3. Дифференциально-трансформаторный магнитный усилитель на дросселях с прямоугольной кривой намагничивания представляет собой 
в принципе симметричный блок дизъюнкции (конъюнкции) на магнитных ключах с выпрямленным выходом.

\section{ЛИ Т Е Р А Т У Р А}

1. С арв В., О явээр М., Саккос Т., Хунт Ю., В сб.: Магнитные преобразователи электроэнергии с удвоением частоты, Таллин, 1972.

2. Л а усм а а Т., Изв. АН ЭССР, Физ. Матем., 20, 188 (1971).

3. Л а у см а а Т., В сб.: Синтез функциональных преобразователей на дросселях с прямоугольной кривой намагничивания. Цепи преобразования параметров электроэнергии, Таллин, 1975.

Ннститут термофизики и электрофизики Академии наук Эстонской ССР

Поступила в редакцию 12/IX 1975

T. LAUSMAA, J. HUNT

TÄISNURKSE MAGNEETIMISKŌVERAGA SUDAMIKEGA DIFERENTSIAAL-TRANSFORMATOORSE MAGNETVOIMENDI ASESKEEM

Esitatud aseskeem, mis võtab arvesse südamike hüstereesi ja dünaamilise takistuse, lihtsustab tunduvalt võimendi arvutamist ning analüüsi, võimaldades ühtlasi paremini mõista tema tööprotsessi olemust.

\section{T. LAUSMAA, J. HUNT}

EQUIVALENT CIRCUIT FOR DIFFERENTIAL-TRANSFORMATIONAL MAGNETIC AMPLIFIER BASED ON TRANSDUCTORS WITH RECTANGULAR HYSTERESIS LOOP

The derived equivalent circuit considerably simplifies the calculation and analysis of the amplifier and gives a better understanding of its operation processes. 